\title{
A case of TBC1D32-related ciliopathy with novel compound heterozygous variants
}

\author{
Ji Ye Ahn ${ }^{1 \oplus}$, Soo Yeon Kim ${ }^{1,2 *} \oplus$, Byung Chan Lim ${ }^{1,2}$, Ki Joong Kim ${ }^{1 \oplus}$, and Jong Hee Chae ${ }^{1,2 \oplus}$ \\ ${ }^{1}$ Department of Pediatrics, Pediatric Neuroscience Center, Seoul National University Children's Hospital, Seoul, Korea \\ ${ }^{2}$ Rare Disease Center, Seoul National University Hospital, Seoul, Korea
}

\begin{abstract}
Primary cilium has a signal transduction function that is essential for brain development, and also determines cell polarity and acts as a mediator for important signaling systems, especially the Sonic Hedgehog (SHH) pathway. TBC1D32 is a ciliary protein, implicated in SHH signaling. Biallelic mutations in the TBC1D32 gene causes a kind of ciliopathy, heterogeneous developmental or degenerative disorders that affect multiple organs, including the brain. Here we report a boy who carried compound heterozygous variants in TBC1D32. The patient showed hypotonia, respiratory difficulty, and multiple anomalies at his birth. He was diagnosed with congenital hypopituitarism and treated with T4, hydrocortisone, and growth hormone. Despite the hormonal replacement, the patient needed long-term respiratory support with tracheostomy and nutritional support with a feeding tube. His developmental milestones were severely retarded. Hydrocephalus and strabismus developed and both required surgery, during the outpatient follow-up. Whole-exome sequencing indicated compound heterozygous variants, c.2200C >T (p.Arg734*) and c.156-1G>T, in TBC1D32 gene. This is the first Korean case of TBC1D32-related ciliopathy and we reported detailed and sequential clinical features. This case demonstrated the utility of whole-exome sequencing and provided valuable clinical data on ultra-rare disease.
\end{abstract}

Key words: TBC1D32, Ciliopathies, Hypopituitarism.

\section{Introduction}

Primary cilium has an important role in sensory and signal transduction and mediating Sonic Hedgehog pathway, which is essential for brain development $[1,2]$. Defects in the primary cilium caused a heterogeneous group of a disorder called ciliopathies [3]. Oral-facial-digital (OFD) syndrome is one of the ciliopathies characterized by major abnormalities of the oral cavity, face, and digits [4]. The TBC1D32 gene, ascertained as a ciliary protein by proteomic analysis, was first reported in a patient with clinically diagnosed OFD syndrome type IX in 2014 [5]. Since then, only 7 patients including familial cases have been reported to have biallelic variants in TBC1D32 [5-8]. They presented diverse phenotypes: midline cleft, micro/anophthalmia, polydactyly, agenesis of corpus callosum, hydrocephalus, or pituitary hypoplasia with hormonal insufficiency. All cases were confirmed by massive parallel sequencing (whole exome sequencing or whole genome sequencing) and indicated its clinical utility on the reports. However, qualified clinical data and following physician's insight will be a critical point for diagnosis,

\footnotetext{
Received: 16 December 2020, Revised: 31 January 2021, Accepted: 3 February 2021, Published: 30 June 2021

${ }^{*}$ Corresponding author: Soo Yeon Kim, M.D. (D) https://orcid.org/0000-0003-2240-3647

Department of Pediatrics, Pediatric Neuroscience Center, Seoul National University Children's Hospital, Rare Disease Center, Seoul National University Hospital, 101 Daehak-ro, Jongno-gu, Seoul 03080, Korea.

Tel: +82-2-2072-4065, Fax: +82-2-2072-3917, E-mail: iidue@naver.com

Conflict of interest: The authors declare that they do not have any conflicts of interest.

(ㄷ) This is an open-access article distributed under the terms of the Creative Commons Attribution Non-Commercial License (http://creativecommons.org/licenses/by-nc/4.0/) which permits unrestricted non-commercial use, distribution, and reproduction in any medium, provided the original work is properly cited.

(c) Copyright 2021 by the Korean Society of Medical Genetics and Genomics 
because some patients could be mistaken for acquired perinatal problems. But previous reports mainly focused on the genetic study and its utility and did not describe detailed and long term clinical information.

Here, we report a patient who carried compound heterozygous variants of TBC1D32 with detailed clinical presentation, summarized by neonatal hypotonia, congenital hypopituitarism, and multiple anomalies followed by severe developmental delay and eye manifestation.

\section{Case}

A 5-day-old boy was admitted to neonatal intensive care unit (NICU) in Seoul National University children's Hospital to evaluate his multiple anomalies and respiratory difficulty. The patient was born at gestational age of 38+1 weeks from healthy non-consanguineous parents after an uneventful pregnancy. At the time of birth, he had no initial crying and his Apgar score
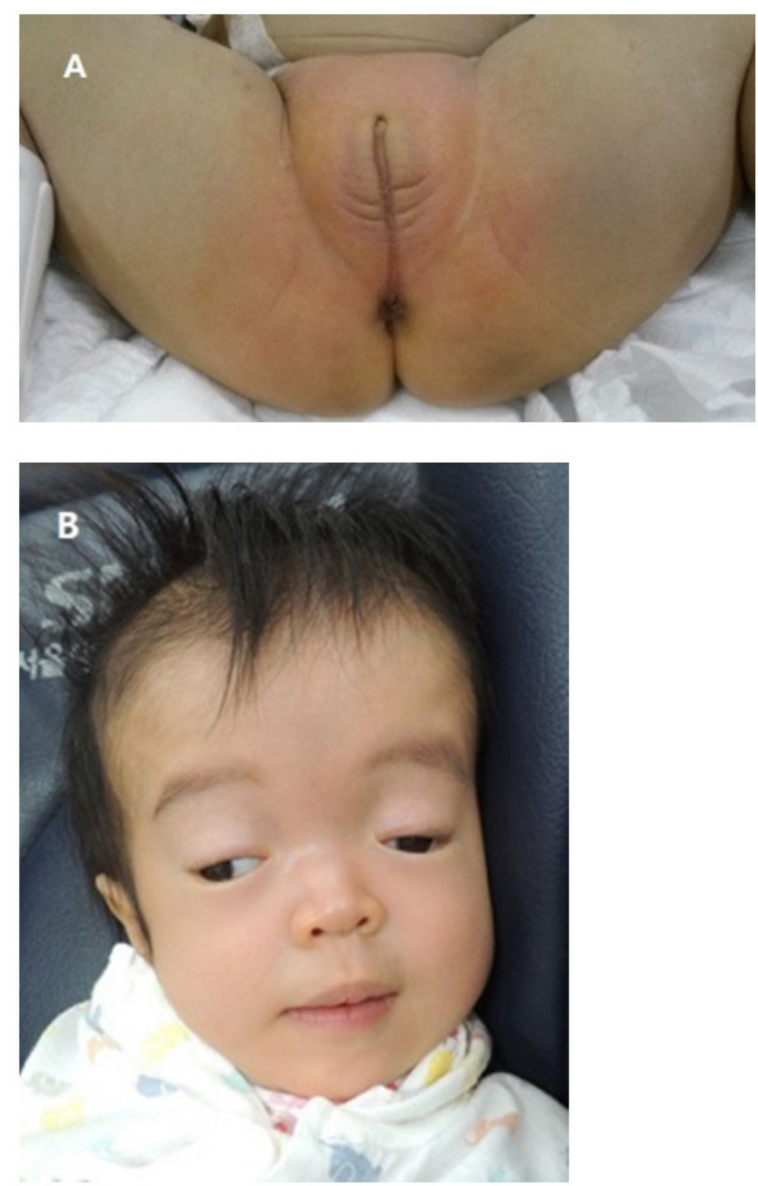

Fig. 1. Clinical photography at admission (A) and 27 months old (B). (A) The patient presented with ambiguous genitalia, perineal ectopic anus. (B) The patient showed a prominent forehead, hypertelorism, bilateral ptosis, strabismus. revealed 2 points at 1 minute, 4 points at 5 minutes. He needed immediate intubation and mechanical ventilation.

His birth weight, height, and head circumference were 3.4 $\mathrm{kg}$ (50th-75th percentile), $47 \mathrm{~cm}$ (3rd-5th percentile), and 30.5 $\mathrm{cm}(<1 \mathrm{st}$ percentile) respectively. He showed a coarse face with hypertelorism and bilateral ptosis. On physical examination, choanal atresia, perineal ectopic anus, ambiguous genitalia, and relatively short limbs were noted (Fig. 1). There were no abnormal findings on neonatal screening test including 17- $\alpha-$ hydroxyprogesterone (17-OHP) and the karyotype was 46, XY. The abdominal ultrasonography revealed bilateral testes in scrotal sacs, instead of female reproductive organs. Clinicians from endocrinology and urology decided patient's sex as male based on test results and family counselling. His blood pressure was constantly low without any triggering factors and responded only to hydrocortisone administration. He was suggested as adrenal insufficiency due to low basal serum adrenocorticotropin (ACTH) of $1 \mathrm{pg} / \mathrm{mL}$ (reference range, $10-60 \mathrm{pg} / \mathrm{mL}$ ) and serum cortisol of $0.1 \mu \mathrm{g} / \mathrm{dL}$ (reference range, $2-11 \mu \mathrm{g} / \mathrm{dL}$ ) at $8 \mathrm{AM}$. Additional hormonal studies were performed and the results were

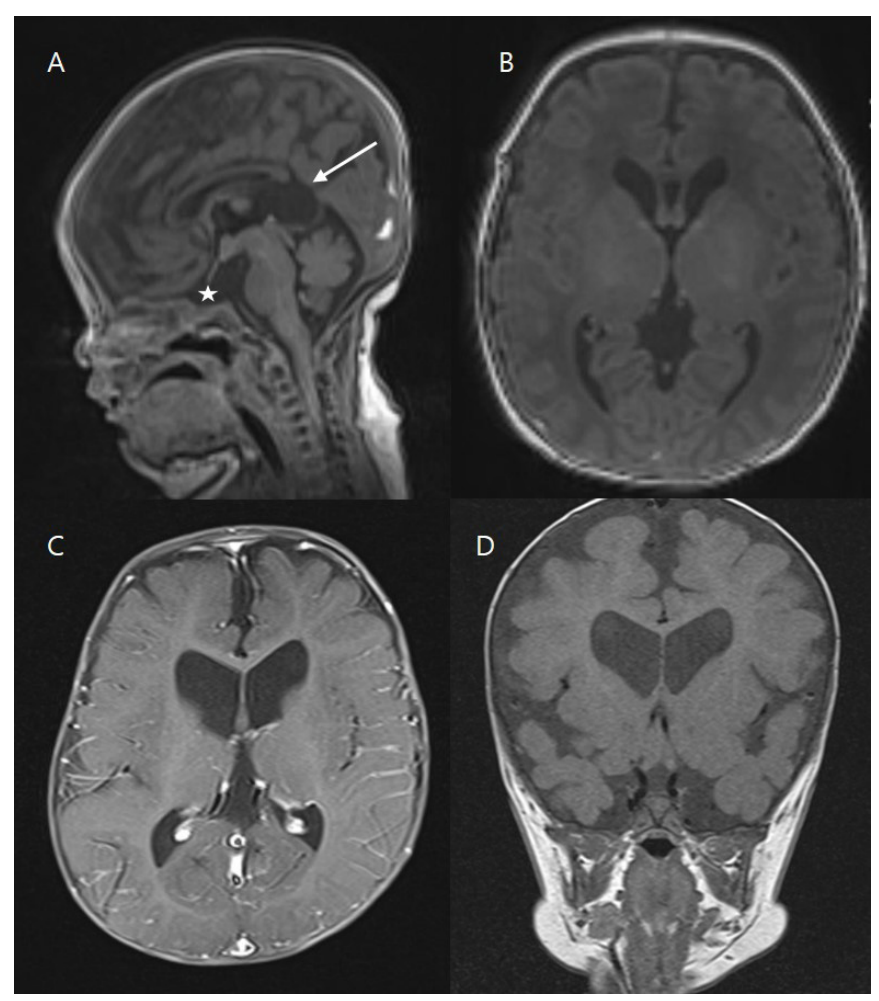

Fig. 2. Brain magnetic resonance imaging showed sagittal $T 1(A)$, axial $\mathrm{T} 1$ (B) at 14 days old and axial T1 (C), coronal T1 (D) at 16 months old. (A) and (B) showed absent pituitary gland (asterisk), partial agenesis of corpus callosum (arrow). (C) and (D) images showed an enlarged lateral ventricle and hydrocephalus compared with images at 14 days old. 
as follows: insulin growth factor 1 (IGF-1) level was below $5 \mathrm{ng} /$ $\mathrm{mL}$ (reference range, $6.6-268.8 \mathrm{ng} / \mathrm{mL}$ ), Thyroid-stimulating hormone (TSH) and free T4 was $2.84 \mu \mathrm{IU} / \mathrm{mL}$ (reference range, $0.4-4.1 \mu \mathrm{lU} / \mathrm{mL}$ ), and $0.16 \mathrm{ng} / \mathrm{dL}$ (reference range, $0.7-1.8 \mathrm{ng} / \mathrm{dL}$ ), respectively. The laboratory test also indicated hypernatremia with persistent high urine output, which suggested diabetes insipidus. Brain magnetic resonance imaging revealed partial agenesis of corpus callosum and no visible pituitary stalk (Fig. 2). So, he was diagnosed with primary panhypopituitarism.

Because his respiratory insufficiency continued after hormonal treatment, he underwent tracheostomy at 26 days of age and was discharged at 3 months old with tracheostomy and feeding tube. He received regular outpatient check-ups after discharge. Hydrocephalus developed and the patient underwent ventriculoperitoneal shunt surgery at 16 months old. Since then, he has undergone correction at 30 months old for hydrocele and inguinal hernia. After 2 years of testosterone treatment for micropenis, he is currently showing male external genitalia. He was also diagnosed with strabismus which required surgery at 23 months old. His developmental milestones were markedly delayed despite constant rehabilitation. He could control his head at 29 months old and rollover at 36 months. At the last follow-up of 6 years old, he could sit up and pointing familiar objects according to his parents' simple commend, but could not say a meaningful word.

He was enrolled in the Korean Undiagnosed Disease Program and had chromosomal microarray as a first-tier test and trio whole-exome sequencing (WES) as a second-tier test. For whole exome sequencing, capture probes targeting the entire exonic regions based on SureSelect Human All Exon V5 (Agilent Technologies, Inc., Santa Clara, CA, USA) were used. Sequencing was performed by HiSeq 2500 System (Illumina, San Diego, CA, USA) with 100 paired-end reads. The reads were aligned to Genome Reference Consortium Human Build 37 using Burrow-Wheeler alignment (v.2.2.0). Detailed analytic protocol was described in our previous study [9]. As a result, compound heterozygous variants in TBC1D32, c.2200C > T (p.Arg734*) and c. 156-1G>T, were identified and segregation test was completed by Sanger sequencing (Fig. 3). Both variants were classified as pathogenic according to the guideline of American College of Medical Genetics and Genomics (ACMG) [10]. Both were nonsense or canonical \pm 1 or 2 splice sites variants in a gene where loss of function is a known disease mechanism (PVS1). They were absent from population databases such as gnomAD and 1000genome (PM2). Computational evidence also supported their deleterious effect (PP3) and each variant was inherited from mother and father, respectively (PM3). Although there was no evident human phenotype with TBC1D32 in Online Mendelian Inheritance in Man (OMIM), we diagnosed him as TBC1D32-related ciliopathy according to a literature review [5].

This study was approved by the institutional review board (IRB) of Seoul National University Hospital (IRB No. 1406-081-588 for whole exome sequencing and review of medical records). His parents provided written informed consent.

\section{Discussion}

This report describes the first Korean case of TBC1D32-related ciliopathy. The index case carried two novel variants, c.2200C>T (p.Arg734*) and c.156-1G>T, both classified as pathogenic according to the ACMG guideline. But we could not confirm the clinical significance because only a single patient without func-

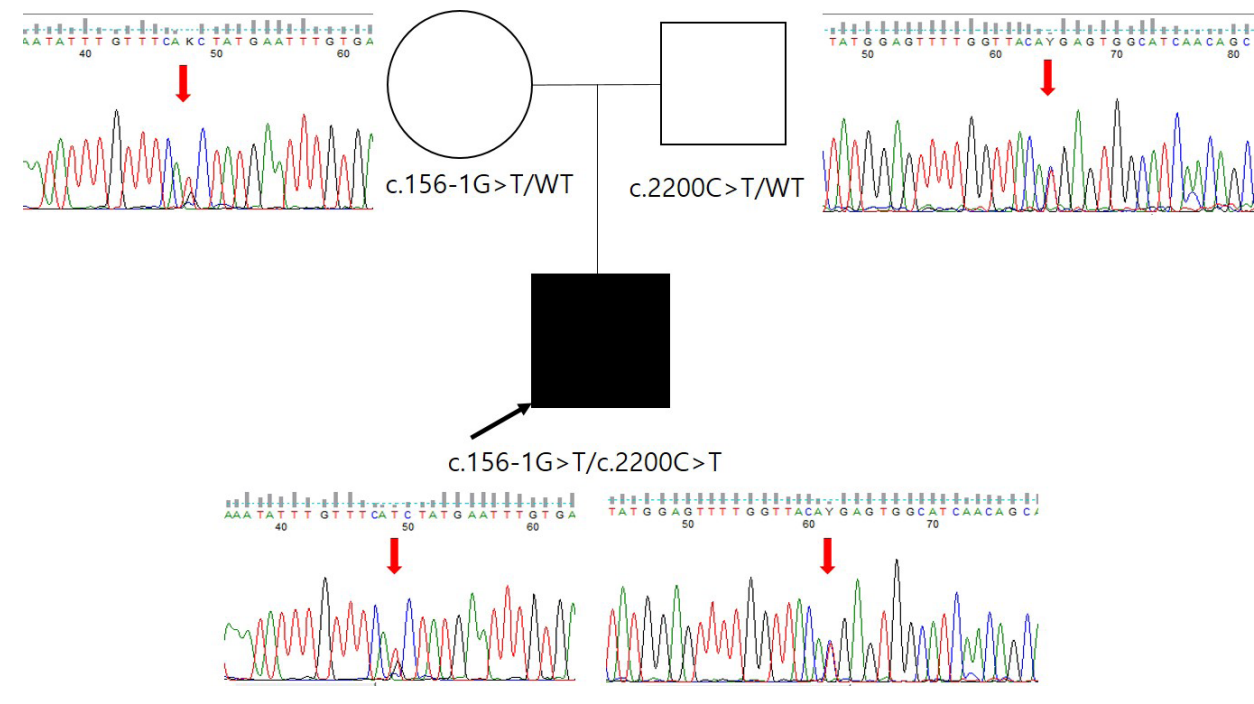

Fig. 3. Pedigree and electropherogram of Sanger sequencing. The patient and his mother share c.156-1G $>$ T mutation, and the patient and his father share c.2200C $>T$ (p. Arg734*) mutation on TBC1D32. The red arrows indicate the genomic position where the base changed. 


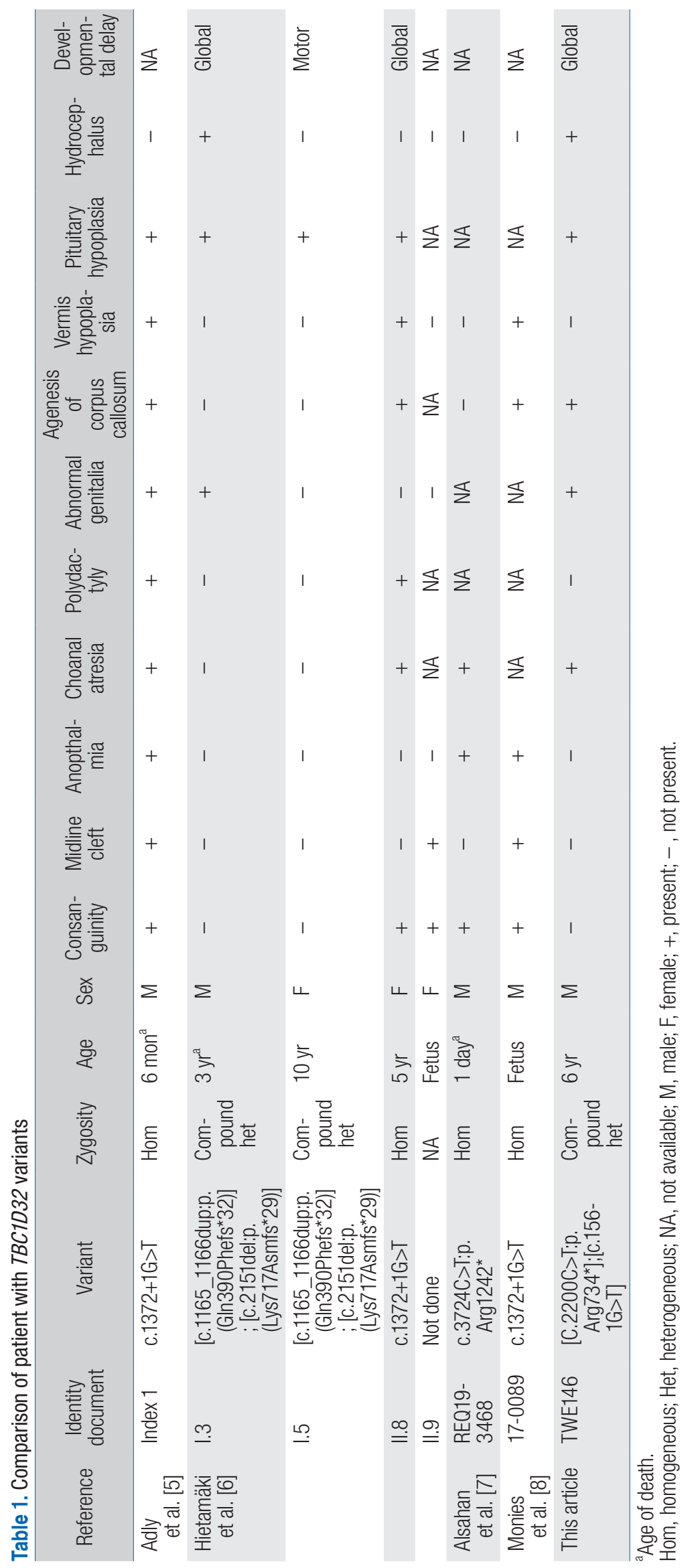


tional validation was reported at the time of data generation [5]. However, we considered the gene as a causative one, based on the patient's phenotyping and literature review first, not on variants themselves [6,11]. Ko et al. [11] revealed that mutant mice exhibit exencephaly with the absence of cephalic ventral midline furrow, poorly developed eyes, and preaxial polydactyly. And we recently convinced his molecular diagnosis as additional clinical reports have been published between 2019 and $2020[6,7]$.

TBC1D32-related ciliopathy has been recently documented only in some case reports and extremely rare. To date, only 7 cases were reported worldwide, including 2 families and 2 fetuses (Table 1) [5-8]. Previously reported patients showed neonatal hypotonia and characteristic features including a prominent forehead, hypertelorism, and low-set ears. Many of them had congenital anomalies such as polydactyly, choanal atresia, and abnormal genitalia, especially in boys. Hormonal insufficiency was quite characteristic findings with the absent or ectopic pituitary gland. Hietamäki et al. [6] reported 2 families with biallelic TBC1D32 variants as syndromic hypopituitarism. These features seemed to be homogeneous, but each manifestation was noted in 50\%-60\% of reported cases, which made the diagnosis challenging. Our case also has characteristic facial features including a prominent forehead, hypertelorism, and low-set ears. He was also diagnosed with panhypopituitarism at neonatal period by multiple hormonal studies. However skeletal anomalies were not evident. There was only few information about patients' neurodevelopmental outcomes. Most of the patients seemed to show motor delay, but 3 patients have died before 3 years old [6]. In the aspect of cognition, a Finnish patient had normal intelligence whereas another patient could not say a word as well as walk independently till 5 years old, similar to our index case [6]. This discrepancy might be originated from perinatal distress because severely retarded patients including our case presented with neonatal hypotonia which requires respiratory support and had NICU care. Not only perinatal distress, but also TBC1D32 protein function depending on the location of the mutation may be affected, but it is difficult to determine due to the lack of reported patients. Among the TBC domain genes, there are other examples of protein structure analysis, but unfortunately, the location and domain of the TBC1D32 protein have not been analyzed for proteomic analysis, so it is difficult to determine the location of the mutation and the abnormality of the phenotype in this case [12]. We think this phenotypic diversity is quite comparable with other types of ciliopathies (e.g. Joubert syndromerelated disease, Bardet-Biedl syndrome) $[13,14]$. For specific genotype-phenotype correlation, further clinical and functional studies are crucial.

In conclusion, we report a Korean boy who carried novel compound heterozygous variants in TBC1D32, a very first case in Korea. The patient showed neonatal hypotonia, congenital hypopituitarism with ambiguous genitalia, and profound developmental delay, but no microphthalmos or hand/feet anomalies. Our case demonstrated a clinical utility of WES in extremely rare genetic disorders, although comprehensive phenotyping would provide an important key to analysis as well as adequate care in practice.

\section{Acknowledgements}

This study was supported by the Research Program funded by the Korea Centers for Disease Control and Prevention (Grant No. 2018-ER6901-02).

\section{Authors' Contributions}

Conception and design: JHC, KJK. Acquisition of data: BCL, JYA. Analysis and interpretation of data: JHC. Drafting the article: JYA. Critical revision of the article: SYK. Final approval of the version to be published: SYK.

\section{References}

1. Guemez-Gamboa A, Coufal NG, Gleeson JG. Primary cilia in the developing and mature brain. Neuron 2014;82:511-21.

2. Nozawa $\mathrm{Y}$, Lin $\mathrm{C}_{1}$ Chuang PT. Hedgehog signaling from the primary cilium to the nucleus: an emerging picture of ciliary localization, trafficking and transduction. Curr Opin Genet Dev 2013;23:429-37.

3. Hildebrandt F, Benzing $T$, Katsanis N. Ciliopathies. N Engl J Med 2011;364:1533-43.

4. Gurrieri F, Franco B, Toriello H, Neri G. Oral-facial-digital syndromes: review and diagnostic guidelines. Am J Med Genet A 2007;143A:3314-23.

5. Adly $N_{1}$ Alhashem A, Ammari A, Alkuraya FS. Ciliary genes TBC1D32/ C6orf170 and SCLT1 are mutated in patients with OFD type IX. Hum Mutat 2014;35:36-40

6. Hietamäki J, Gregory LC, Ayoub S, livonen AP, Vaaralahti K, Liu X, et al. Loss-of-function variants in TBC1D32 underlie syndromic hypopituitarism. J Clin Endocrinol Metab 2020;105:1748-58.

7. Alsahan N, Alkuraya FS. Confirming TBC1D32-related ciliopathy in humans. Am J Med Genet A 2020;182:1985-7.

8. Monies D, Abouelhoda M, Assoum M, Moghrabi N, Rafiullah R, Almontashiri $N_{1}$ et al. Lessons learned from large-scale, first-tier clinical 
exome sequencing in a highly consanguineous population. Am J Hum Genet 2019;104:1182-201.

9. Kim SY, Jang SS, Kim H, Hwang H, Choi JE, Chae JH, et al. Genetic diagnosis of infantile-onset epilepsy in the clinic: application of wholeexome sequencing following epilepsy gene panel testing. Clin Genet 2021;99:418-24.

10. Richards S, Aziz N, Bale S, Bick D, Das S, Gastier-Foster J, et al.; ACMG Laboratory Quality Assurance Committee. Standards and guidelines for the interpretation of sequence variants: a joint consensus recommendation of the American College of Medical Genetics and Genomics and the Association for Molecular Pathology. Genet Med
2015;17:405-24.

11. Ko HW, Norman RX, Tran J, Fuller KP, Fukuda M, Eggenschwiler JT. Broad-minded links cell cycle-related kinase to cilia assembly and hedgehog signal transduction. Dev Cell 2010;18:237-47.

12. Balestrini $S$, Milh $M$, Castiglioni $C$, Lüthy $K$, Finelli MJ, Verstreken $P$, et al. TBC1D24 genotype-phenotype correlation: epilepsies and other neurologic features. Neurology 2016;87:77-85.

13. Brancati F, Dallapiccola B, Valente EM. Joubert syndrome and related disorders. Orphanet J Rare Dis 2010;5:20.

14. Suspitsin EN, Imyanitov EN. Bardet-Biedl syndrome. Mol Syndromol 2016;7:62-71. 